\title{
FABRICATION OF DOUBLE-SIDED MICROFLUIDIC STRUCTURES VIA 3D PRINTED TRANSFER MOLDING
}

\author{
Casey C. Glick, Mitchell T. Srimongkol, Aaron Schwartz, William Zhuang, Joseph Lin, Roseanne Warren, \\ Dennis Tekell, Panitan Satimalee, Judy Kim, Caroline Su, Kyungna Kim, and Liwei Lin \\ University of California, Berkeley, USA
}

\begin{abstract}
In this work, we demonstrate the use of 3D printed molds for rapidly fabricating multi-layer PDMS-based microfluidic devices. Because $3 \mathrm{D}$ printing allows for versatile and cost-effective mold construction, it can produce significantly more varied features than those generated by soft lithography. We first discuss adaptations to single-layer 3D molding, including a glass bonding technique to compensate for the limitations of surface roughness and $0.55 \mathrm{~mm}$ built-in inlet and outlet ports to eliminate fabrication steps. Next we introduce two-sided fabrication methods, facilitated by novel built-in alignment marks. These techniques allow the construction of formerly difficult to achieve features such as non-planar $350 \mu \mathrm{m}$ membranes, used to fabricate a single-layer membrane valve which actuates at 200kPa, and single-layer microfluidic vias, used to generate 3D flow patterns. Lastly, we demonstrate an intra-layer bonding technique where a custom 3D printed stamp selectively applies liquid PDMS adhesive, compensating for surface roughness while preventing channel clogging. Together, these techniques enable the rapid assembly of multi-layer PDMS-based microfluidic devices, combining the versatility and speed of emerging 3D printing technology with the known mechanical and biological properties of PDMS favored by microfluidic researchers.
\end{abstract}

\section{INTRODUCTION}

Microfluidics has rapidly advanced in the fields of chemical and biological research, commonly known as Lab-on-a-Chip (LoC), since 1980 s due to its unique ability to make low-cost, highthroughput platforms [1-3]. The most far-reaching breakthrough in microfluidics has been the development of soft-lithography - using rigid micromachined molds to shape elastomeric polymers. Among the polymeric materials, Poly(dimethylsiloxane) (PDMS) is commonly used due to its numerous ideal properties, including its low cost, strength, transparency, and especially biocompatibility [4].

Traditional methods for fabricating microfluidic devices involve photolithography to construct micro-molds with very fine features; however, the process can be lengthy and costly. Additionally, soft lithography is limited to rectilinear features constructed through additive micromachining processes. [5] For example, while circular channels are common in large fluidic systems and are beneficial for microfluidic devices with internal movable components, [6] to date few groups have developed techniques for circular channels in microfluidics. [7]. The increasing demand for microfluidic devices is particularly high for double and multi-layered devices to allow for the implementation of more sophisticated structures and components (e.g. valves, pumps, and other active control mechanisms). Although multi-layer PDMS manufacturing techniques have been demonstrated, they are often time consuming, labor-intensive, and inaccurate.

$3 \mathrm{D}$ printing has presented a unique route to build multi-layer microfluidic devices directly or indirectly with the additional molding process. For example, various groups have used 3D printers previously to make simple microfluidic devices with complex and truly 3D geometries, including microfluidic devices without moving elements, such as resistors, [8] mixers, modular components, [9] and microfluidic devices with movable components, such as capacitors, diodes and transistors. [10] Although 3D printed microfluidic devices are currently limited by: (1) the available resolution of the printer; (2) surface roughness; [11] and (3) material types, [12] the rapid developments of the $3 \mathrm{D}$ printing technologies are expected to advance and address these matters in the coming years. Additionally, while direct 3D printing is a rapid process for prototyping, for making multiple copies of microfluidic devices, it is slower, more expensive, and less reliable than transfer molding performed by pouring polymer into a mold. Thus, we present a hybrid approach to combine $3 \mathrm{D}$ printing with molding.

This work advances the $3 \mathrm{D}$ printed transfer molding technique from single-sided microfluidics $[13,14]$ to multi-layer microfluidic manufacturing, utilizing the ease of $3 \mathrm{D}$ printing to create multiple molds with alignment structures to shape multiple layers of PDMS structures and quickly assemble them at the end. Using this molding method, we create complex geometries in PDMS, including vias, thin membranes, and rounded channels and demonstrate rapid assembly of multi-layer microfluidic devices using built-in alignment marks which allow precise positioning of each layer without the need for a microscope.

\section{EXPERIMENTAL TECHNIQUES 3D printed molds}

Microfluidic devices were designed and converted from a positive to a negative mold shape using the computer-aided design program SolidWorks. 3D printing of molds was achieved using a Projet 3000 3D printer. During printing, the Projet 3000 deposited layers of structural epoxy (VisiJet ${ }^{\circledR}$ EX200 plastic material) and sacrificial wax (VisiJet ${ }^{\circledR}$ S100 support material); the wax was used as a temporary support for hollow spaces as well as to provide a foundational layer for the mold, and was removed during postprocessing. [15]

\section{Mold post-processing}

Following printing, the molds were cleaned to remove the sacrificial wax. First, the molds were baked in a VWR $1330 \mathrm{FM}$ oven $75^{\circ} \mathrm{C}$ for $45 \mathrm{~min}$ to melt the sacrificial wax. The molds were then washed in a sequence of three cleaning baths for $10 \mathrm{~min}$ in each bath to remove leftover wax: warm Bayes mineral oil, Ajax dish detergent in water, and potable water. The baths were heated to $75^{\circ} \mathrm{C}$ to ensure the wax did not re-solidify, and were placed on a hotplate with a magnetic stir bar to enhance removal of wax, oil, and soap, respectively. The molds were then dried and residual water was removed by baking the molds at $80^{\circ} \mathrm{C}$ for $60 \mathrm{~min}$. After cleaning and drying, the $3 \mathrm{D}$ printed molds were treated with an anti-adhesive agent (Trichloro( $1 H, 1 H, 2 H, 2 H$-perfluorooctyl)silane (PFOTS), Sigma Aldrich) gas, making the surface hydrophobic to facilitate the rapid removal of PDMS. [16] The molds were placed in vacuum desiccator with 10 drops of PFOTS agent for $30 \mathrm{~min}$. Shorter times resulted in PDMS bonding to the mold and longer times caused a build-up of PFOTS which inhibited complete curing of the PDMS near the surface. $[11,17]$

\section{PDMS molding}

The $3 \mathrm{D}$ printed molds were placed onto a foil-wrapped 3D printed molding tray, which reduces PDMS waste. PDMS (Sylgard 
184 Elastomer Kit) was prepared using the standard 10:1 as the base:curing agent ratio. The PDMS mixture was degassed in a vacuum chamber for 10 minutes and then poured into the 3D printed molds. The filled molds were then placed in the vacuum chamber for $45 \mathrm{~min}$ to degas and increase PDMS conformity. Following the degassing, the molds were baked in an $80{ }^{\circ} \mathrm{C}$ oven for $50 \mathrm{~min}$. The PDMS microfluidic devices were removed from the molds by first cutting away excess PDMS and then by manually peeling the PDMS loose from the mold. The finer the printed features, the more carefully this removal must be performed. Provided no features have broken during the de-molding process and PDMS did not permanently bond to the mold, the molds are reusable without an additional cleaning process. If during de-molding PDMS adheres excessively to the $3 \mathrm{D}$ printed mold, the hydrophobicity of the molds must be 'recharged' by repeating the PFOTS treatment (approximately every 10-20 moldings).

\section{RESULTS AND DISCUSSION}

Figure 1 illustrates the process flow for fabricating PDMS microfluidic devices using 3D printed molds. In this example, three microchannels with elliptical-shape reservoirs of dimensions $1.5 \times 5.9 \mathrm{~mm}^{2}, 1.1 \times 5.9 \mathrm{~mm}^{2}$ and $0.7 \times 5.9 \mathrm{~mm}^{2}$ were designed as shown in Fig. 1a. The overall dimension of the mold was 20x20x2 $\mathrm{mm}^{3}$ with quarter-circle $(\mathrm{R}=2.5 \mathrm{~mm})$ pillars at the four corners facilitating removal of the PDMS after curing. Each channel was constructed with built-in fluid inlets and outlets molded from pillars $0.55 \mathrm{~mm}$ in diameter and $5 \mathrm{~mm}$ in height. The inlet pillars were strengthened by widening them halfway down to $1 \mathrm{~mm}$ in diameter.
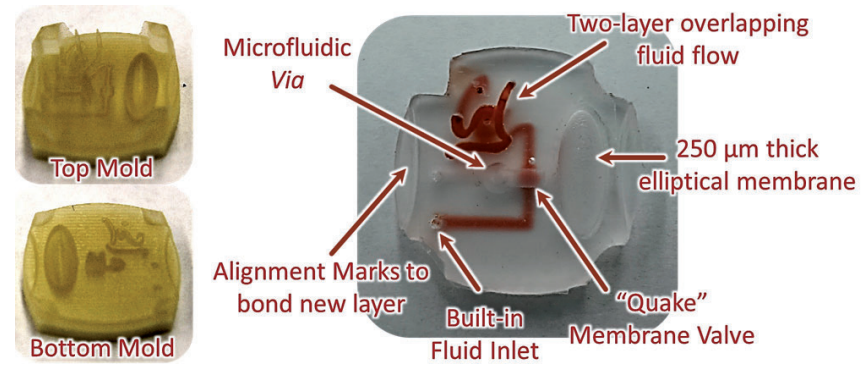

Figure 1. Illustration of the $3 D$ printed transfer molding technique and process. The microfluidic device mold is designed using CAD and the PDMS structure can be bonded onto a glass substrate to make the enclosed channels and reservoirs system. The complex multi-level microfluidic device is fabricated using the $3 D$ printed mold shown, and includes two layers of overlapping fluid flow, an elliptical membrane, a "Quake" membrane value, multiple microfluidic vias, built-in fluid inlets, and alignment marks to create a stacked, multi-layer device.

The device mold was fabricated via the $3 \mathrm{D}$ printing process and PDMS was applied, cured and released from the mold by means of the PDMS molding steps described in the Experimental section. Built-in fluid inlets can be easily incorporated to the device through the mold design, further simplifying fabrication by eliminating the need for a hole-punching step. These built-in inlets were reliable to pressures of 4 ATM. Narrower inlets were shown to hold against higher opposing pressures as the channel inlet gripped the couple more strongly. However, narrower inlets faced trade-offs with the fragility of the 3D printed mold, breaking during post-processing.

The PDMS device was then bonded to a glass slide to create closed microfluidic channels. Clearly, this 3D printed transfer molding and bonding technique can be used to fabricate conventional microfluidic circuit devices commonly produced by the soft lithography methods with the following advantages: faster and less complex process steps; easy to create complex 3D geometries; ability to fabricate circular channel cross-sections; and built-in microfluidic connectors. For multi-layer devices, a PDMSPDMS bonding technique has been developed with a 3D printed stamp as well as the use of built-in alignment marks for rapid assembly of more than one layer of prototype PDMS structures in this work without a microscope. These techniques make possible the design, fabrication and assembly of a complex microfluidic system as shown in Fig. 1. For these more complex devices, the basic procedure of generating a CAD model, 3D printed mold, and PDMS replica of the mold remains the same, for a complex double-sided device incorporating a variety of microfluidic device features.

\section{Single-sided molding}

Using 3D printed molds, semi-circular and fully circular channel geometries are easily fabricated (Fig. 2). Surface texture in the channels arises from the interface between sequential rows of epoxy deposited during the 3D printing step. Figure 2d shows the PDMS device after release from the 3D printed molds, with a closeup view of the device surface. The average surface asperity of the PDMS microfluidic devices in this work, as measured by surface profilometry is approximately $20 \mu \mathrm{m}$ (Fig. 2d). This value is comparable to microfluidic devices fabricated directly by 3D printing, although transfer molded PDMS devices can exhibit significantly smaller channel diameters. Additionally, surface roughness in $3 \mathrm{D}$ printed devices is higher than in devices fabricated by conventional soft lithography, although this feature resolution problem is expected to be overcome as $3 \mathrm{D}$ printers improve. $[18,19]$

Due to the surface roughness of the $3 \mathrm{D}$ printed molds, it is difficult to achieve a tight seal when bonding PDMS and glass using standard techniques such as oxygen plasma and ozone surface treatments. For this reason, specialized bonding techniques are needed to create close-channel microfluidic devices with the 3D printed transfer molding process. Some surface roughness can be reduced by performing a standard surface treatment such as oxygen plasma and then tightly clamping the two components together, mechanically compressing surface profile. However, this technique is unreliable and often causes the glass to break during PDMS-glass bonding. A more reliable technique uses uncured PDMS as both bonding agent and as filler which overcomes drawbacks associated with surface roughness from 3D printing. The method, involves spin coating a layer of PDMS onto a glass slide to achieve a solid bond between the PDMS device and glass slide (Fig. 2f). First, a thin layer of PDMS was spin-coated onto a $20 \times 20 \mathrm{~mm}^{2}$ glass slide (1800RPM). Next, the slide was placed onto a $95^{\circ} \mathrm{C}$ hot plate for one minute to allow the PDMS to partially cure, and then the cured PDMS component was pressed firmly into the still-curing PDMS and held for one minute. Finally, the pressure was released and the device cured for an additional five minutes. The PDMS was held steady during this bonding to prevent slippage.

Figure 2 shows images of fully rounded microfluidic channels fabricated using the $3 \mathrm{D}$ printed transfer molding process. The channels are built by bonding two semi-circular channel PDMS devices. Using built-in alignment marks, the top and bottom PDMS layers can be easily assembled without a microscope (Fig. 2b). Fully rounded fluidic channels may have a specific use in optofluidic lithography. [18] During device manufacture and curing, features contract, allowing components to freely move. However, when used in conjunction with channels with uniform height, this lubricating fluid gap also prevents a tight seal, causing leakage within fluidic diodes or transistor-like components. Due to the physics of thin film fluid flow, there is a direct trade-off between speed of optofluidic feature movement under pressure-driven flow and the rate of fluid leakage, which may be alleviated by 3D molding. 
a

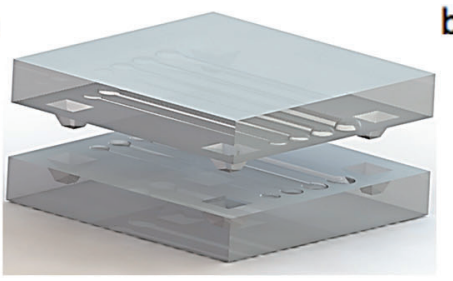

b

\section{PDMS-PDMS Alignment}

d

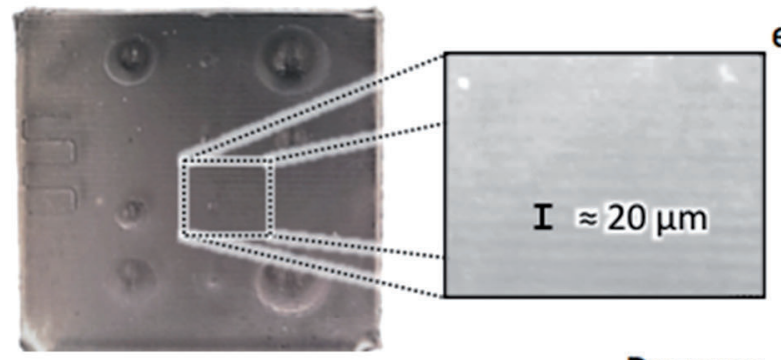

Pressure

f
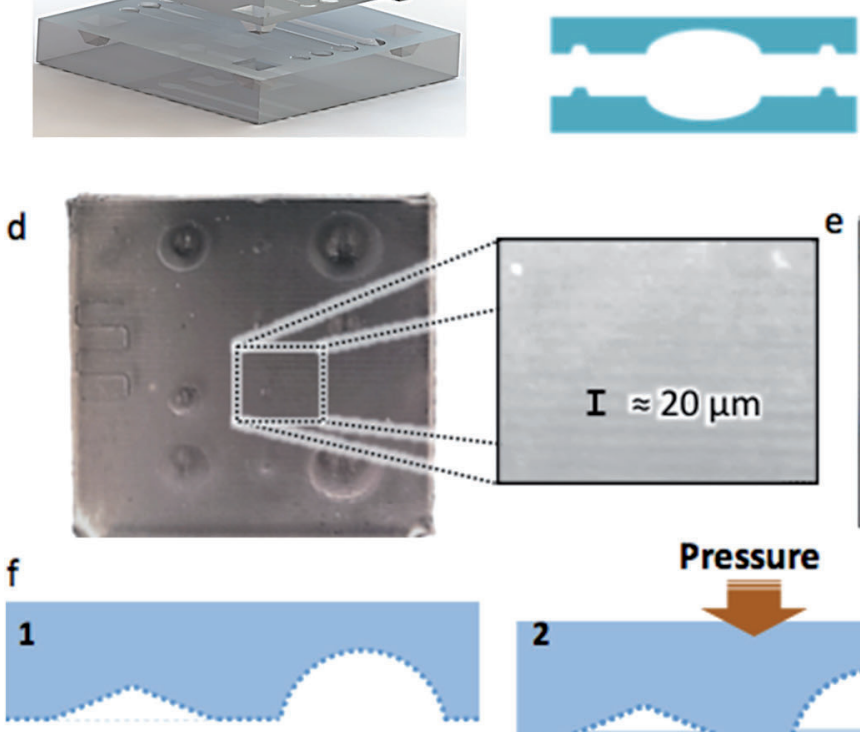

c
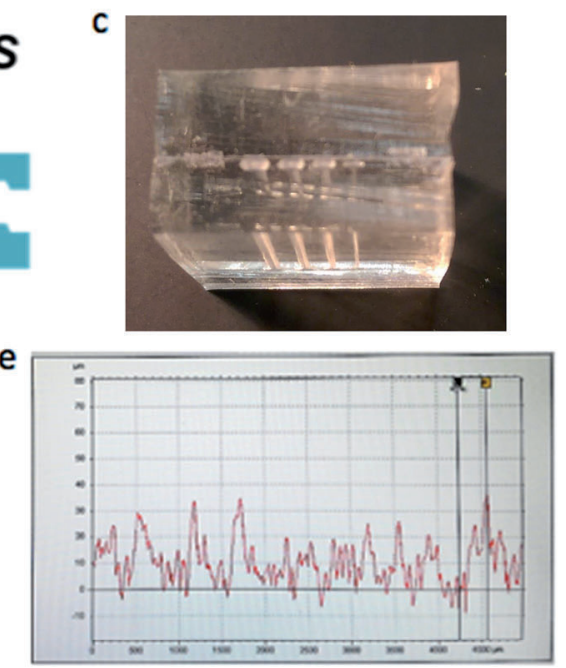
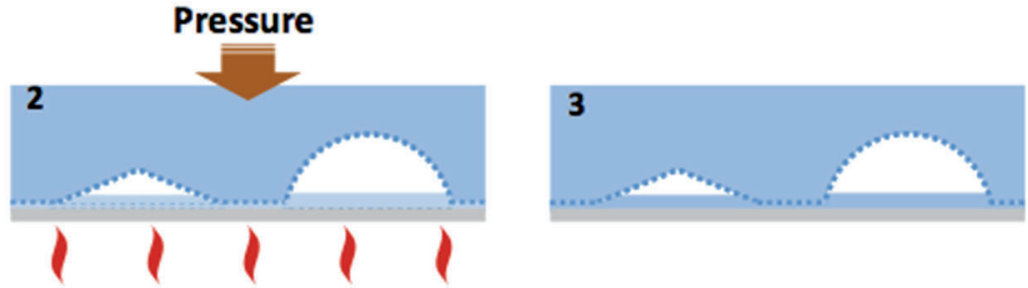

Figure 2. Fabrication capabilities of PDMS microfluidic devices achieved using single-sided molding with a $3 D$ printed template. (a) Photograph of a device assembly process with circular channel geometries. (b) Conceptual illustration of the use of built-in alignment marks for circular channel assembly. (c) Photograph of a device with a circular channel cross-section. (d) Photograph of surface of molded PDMS with zoomed inset, and (e) Profilometer measurement of the mold surface. (f) Process for PDMS-Glass bonding of 3D transfer molded PDMS.

\section{MULTI-LAYER DEVICES}

The increasing demand for microfluidic devices is particularly high for multi-layered devices. [13,14,20,21] Devices with more than one layer allow for the implementation of far more sophisticated and useful structures/components, such as valves or pumps, within the device as well as eliminating geometrical constraints by enabling fluidic detours and vias. Creating conventional multilayer microfluidic devices requires at least two lithography steps and one PDMS-PDMS bonding step (e.g. for "Quake" membrane valves) [22] and up to four lithography steps and three PDMS-PDMS bonding steps (e.g. for PDMS-based fluidic transistors).

Multi-layer construction is used to make a multiple layer device primarily to overcome the limits of 2D traditional microfluidic systems and give a traveling fluid within a device an extra dimension to travel in. In addition, with 3D printed molding, rapid assembly of multilayer microfluidic devices is easily achievable through the use of built-in alignment marks. Alignment marks can be used on the molds, PDMS microdevices, or combinations of the two, making several fabrication techniques possible (see Supporting Information for more detailed descriptions of mold-mold, PDMS-mold, and PDMS-PDMS alignment marks). Alignment marks enable precise positioning of each layer without the need for a microscope.

\section{Thin Membranes and Vias}

With the 3D printed molding approach, complex microfluidic structures including thin membranes and vias can be easily achieved using two-sided molding (Fig. 3a). By creating mold features which nest between top and bottom molds, an empty region can be made which is filled by PDMS during vacuum degassing. This work was able to consistently demonstrate membranes down to $200 \mu \mathrm{m}$ thick. Below that, surface interaction effects (possibly lack of oxygen) [11] interfered with PDMS curing.

Additionally, two-sided molding techniques may be adapted by using with columns that run from the bottom mold and fit into the upper mold, thereby constructing intra-layer fluidic channels. These fluidic vias allow fluid to flow between the top and bottom face of a single layer of PDMS (Fig. 3d). Vias reduce the complexity of multilayer manufacture by allowing three dimensional channel detours, meaning that channels may only require a limited fluidic path to overcome obstacles, such as other microfluidic channels.

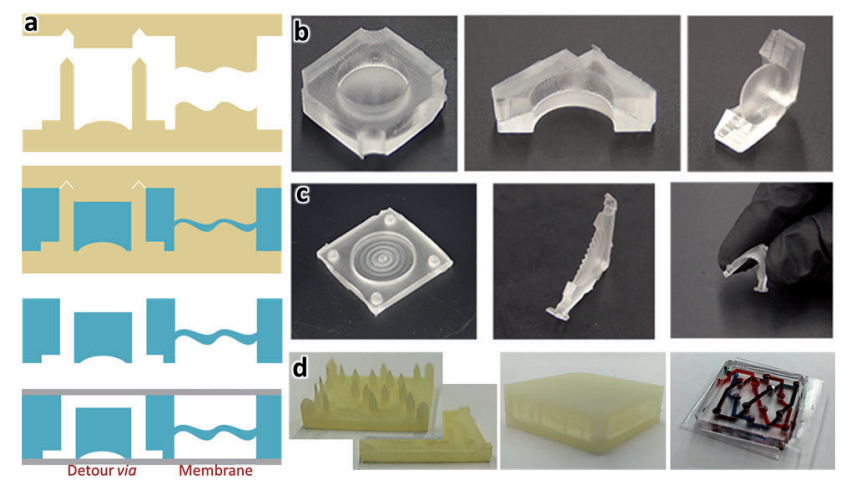

Figure 3. Two-sided molding techniques: vias and thin-film membranes. (a) Fabrication flow for two-sided microfluidic device, including via detour and arbitrary thin membrane, formed by molding on both sides of PDMS. (b) $350 \mu \mathrm{m}$ domed membrane and (c) $350 \mu \mathrm{m}$ sinusoidal membrane. (d) Abbreviated fabrication process for Celtic knot-inspired device with sixteen fluidic vias. 


\section{Membrane Valve}

Another common use for multilayer microfluidic devices is in membrane valves, which use pneumatic or hydraulic pressure in one fluid layer to moderate fluid flow in a secondary layer. [6] Commonly, these membrane (or "Quake") valves are multi-layer constructions which use a number of pneumatic inputs to control complex arrays of microfluidic reactors. Specifically, these membrane valves require two lithographic manufacture steps (lower, and control layers), a spin-coated thin layer of PDMS (the membrane layer) and one alignment step between the upper lower layers. In this work, we fabricated a Membrane Valve using a single-stage double-sided microfluidic molding technique, by linking a detour via with a thin membrane in an upper layer (Fig. 4). The membrane was $350 \mu \mathrm{m}$ thick, and the lower channel was $500 \mu \mathrm{m}$ thick (Fig. 4b). Standard membrane valves need a photoresist reflow step during manufacture to allow the bottom layer to close fully, we were able to implement a rounded lower channel directly from the CAD file. To characterize the closing behavior of membrane valve, we ran a Gate pressure parametric sweep (Fluigent MAESFLO ${ }^{\circledR}$ ) and measured the resulting sourcedrain flow rate (Fluigent Flow Unit L). We found that the valve began closing at $160 \mathrm{KPa}$ and fully closed by $220 \mathrm{KPa}$, and had a mostly linear response during the transition (Fig. 4d)

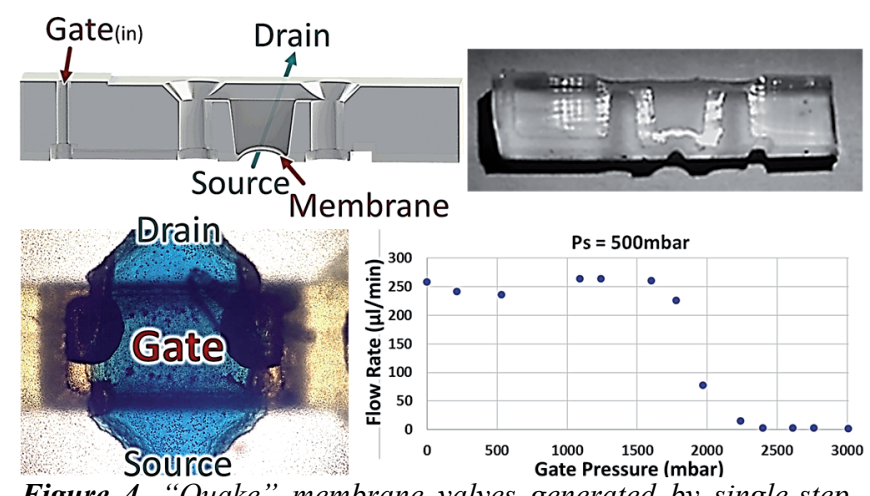

Figure 4. "Quake" membrane valves generated by single-step double-sided molding procedure. (a) Conceptual and (b) crosssectional view of membrane valve. (c) Microscope image illustrating the active valve region. (d) Valve characteristic curves under parametric gate pressure sweep.

\section{CONCLUSION}

Rapid assembly methods for polymer microfluidic devices fabricated using 3D printed technology were presented, where 3D printed molds were used to manufacture components made out of PDMS. With these design features, the number of fabrication techniques possible to manufacture was expanded. In addition to alignment marks, which were used to optimize the bonding procedures during the fabrication of the devices, features such as thin membranes can easily be made. Because the size of the membrane can vary with the expanded resolution design limits of the $3 \mathrm{D}$ printer, the list of purposes a microfluidic device can perform with the feature are expanded. Bonding procedures were also investigated with the $3 \mathrm{D}$ printed molds and procedures such as PDMS-Glass and PDMS-PDMS bonding were evaluated. Bonding applications such as the double-sided channels and multilayer construction were explored in order to measure the effectiveness of the bonding procedures. With 3D printing, molding and bonding of devices can be completed in under a couple hours, and the possibilities of different features and purposes for a microfluidic device are expanded as resolution of features are limited to sizes around $50 \mu \mathrm{m}$.

\section{ACKNOWLEDGMENTS}

The authors would like to thank Xining Zang, Kyungna Kim, and Ariana Moini for their help with this project. Additionally, we thank the CiBER at UC Berkeley and to the NSF GRFP and BSAC for helping fund this project. Travel support has been generously provided by the Transducer Research Foundation.

\section{REFERENCES}

[1] Xia, Y. \& Whitesides, G. Soft lithography. Annual Review Of Materials Science 28, 153-184 (1998).

[2] Duffy, D., McDonald, J., Schueller, O. \& Whitesides, G. Rapid prototyping of microfluidic systems in poly(dimethylsiloxane). Analytical Chemistry 70, 4974-4984 (1998).

[3] Whitesides, G. M., Ostuni, E., Takayama, S., Jiang, X. \& Ingber, D. E.Soft lithography in biology and biochemistry. Annual review of biomedical engineering 3, 335-373 (2001).

[4] Kim, P. et al. Soft lithography for microfluidics: a review. BioChip Journal 2, 1-11 (2008).

[5] Li, X. et al. Desktop aligner for fabrication of multilayer microfluidic devices. Review of Scientific Instruments 86 (2015).

[6] Thorsen, T., Maerkl, S. \& Quake, S. Microfluidic large-scale integration. Science 298, 580-584 (2002).

[7] Abdelgawad, M. et al. A fast and simple method to fabricate circular microchannels in polydimethylsiloxane (PDMS). Lab on a chip 11, 545551 (2011).

[8] Au, A. K., Lee, W. \& Folch, A. Mail-Order Microfluidics: Evaluation of Stereolithography for the Production of Microfluidic Devices. Lab on a Chip 14, 1294-1301 (2014).

[9] Bhargava, K. C., Thompson, B. \& Malmstadt, N. Discrete elements for 3D microfluidics. Proceedings of the National Academy of Sciences 111, 15013-15018 (2014).

[10] Sochol, R. D. et al. 3D printed microfluidic circuitry via multijetbased additive manufacturing. Lab Chip - (2016).

[11] Hwang, Y., Paydar, O. H. \& Candler, R. N. 3D printed molds for nonplanar PDMS microfluidic channels. Sensors and Actuators A: Physical 226, 137-142 (2015).

[12] Wu, S., Yang, C., Hsu, W. \& Lin, L. 3D-printed microelectronics for integrated circuitry and passive wireless sensors. Microsystems \& Nanoengineering 1, 1-9 (2015).

[13] Comina, G., Suska, A. \& Filippini, D. PDMS lab-on-a-chip fabrication using 3D printed templates. Lab Chip 14, 424-430 (2014).

[14] Comina, G., Suska, A. \& Filippini, D. 3D Printed Unibody Labon-a-Chip: Features Survey and Check-Valves Integration. Micromachines 6, 437-451 (2015).

[15] 3D Systems. ProJet TM HD 3000 Technical Specifications (2009).

[16] Stroock, A. D. \& Whitesides, G. M. Components for integrated poly (dimethylsiloxane) microfluidic systems. Electrophoresis 23, 3461-3473 (2002).

[17] Xia, Y. N. \& Whitesides, G. M. Soft lithography. Angewandte Chemie-International Edition 37, 551-575 (1998).

[18] Glick, C. et al. Single-layer microfluidic current source via Optofluidic lithography. MEMS, $201528^{\text {th }}$ IEEE International Conference on, 551-554 (IEEE, 2015).

[19] Glick, C. C. et al. Pressure gain in single-layer microfluidics devices via optofluidic lithography. In Transducers \& Eurosensors XXVII, 404-407 (IEEE, 2013).

[20] Unger, M., Chou, H., Thorsen, T., Scherer, A. \& Quake, S. Monolithic microfabricated valves and pumps by multilayer soft lithography. Science 288, 113-116 (2000).

[21] 31 Weaver, J. A., Melin, J., Stark, D., Quake, S. R. \& Horowitz, M. Static control logic for microfluidic devices using pressuregain valves. Nature Physics 6, 218-223 (2010).

[22] Mosadegh, B. et al. Integrated elastomeric components for autonomous regulation of sequential and oscillatory flow switching in microfluidic devices. Nature physics 6, 433-437 (2010). 